\title{
P59 Marked Differences in Cerebral Haemodynamics Obtained with Transcranial Doppler vs. 2-D Angle-corrected Ultrasound
}

Christopher Brown*, Mahfoudha Al Shezawi, Laura Watkeys, Maggie Munnery, Christopher Pugh, Eric Stöhr, Barry McDonnell

Cardiff Metropolitan University, Cardiff, UK

\section{ABSTRACT}

Introduction: The assessment of middle cerebral artery (MCA) haemodynamics is essential for the diagnosis and monitoring of cerebrovascular disease. However, conventional transcranial Doppler (TCD) may not capture the correct flow velocities because of sub-optimal angles of insonation. Conversely, 2-D ultrasound (2D-US) allows for the visualisation and angle-correction of MCA haemodynamics. Therefore, this study aimed to determine potential differences in MCA haemodynamics obtained with TCD and 2D-US.

Methods: MCA haemodynamics were obtained in a blinded, randomised order with TCD and 2D-US (non-angle-corrected $=2 \mathrm{D}$-US-NON and angle-corrected $=2 \mathrm{D}$-US-ANGLE) from the temporal left posterior window in twenty-seven healthy participants. Recordings were analysed for peak-systolic velocity (PSV), end-diastolic velocity (ED), pulsatility index (PI) and resistance index (RI). Statistical agreements between TCD and 2D-US were determined using linear regression, independent samples $t$-test and Bland-Altman analysis.

Results: MCA haemodynamics obtained with TCD explained less than 50\% of the values obtained with 2D-US-NON \& 2D-US-ANGLE, respectively (PSV $r^{2}=0.34 \& 0.37$; ED: $r^{2}=0.37 \& 0.44$; PI: $r^{2}=0.20 \& 0.22$; RI: $r^{2}=0.30 \& 0.32$ ). Compared with 2D-US-NON, TCD produced similar PSV $(p=0.65)$ but significantly higher ED $(p<0.0001)$, lower PI $(p<0.0001)$ and lower RI $(p<0.0001)$. 2D-US angle-correction resulted in a significantly higher PSV compared with TCD $(p=0.005)$ while all other differences remained. Bland-Altman analysis revealed a bias between the two methods ranging from $11-40 \%$, with large individual variability.

Conclusion: TCD and 2D ultrasound produce significantly different values for MCA haemodynamics, even when 2D-US is non-angle-corrected. This may have important implications when using indices of MCA haemodynamics in the evaluation of cerebrovascular disease.

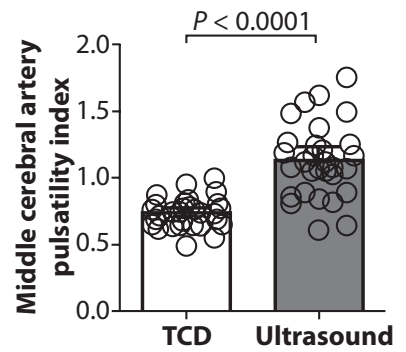

(c) 2019 Association for Research into Arterial Structure and Physiology. Publishing services by Atlantis Press International B.V. This is an open access article distributed under the CC BY-NC 4.0 license (http://creativecommons.org/licenses/by-nc/4.0/). 